\title{
Reversal of the optical force in a plasmonic trap
}

\author{
Lina Huang* and Olivier J. F. Martin \\ Nanophotonics and Metrology Laboratory, Swiss Federal Institute of Technology Lausanne (EPFL), \\ EPFL-STI-NAM, ELG Station 11, CH-1015 Lausanne, Switzerland \\ *Corresponding author: lina.huang@epfl.ch
}

Received September 17, 2008; accepted October 20, 2008;

posted November 11, 2008 (Doc. ID 101636); published December 12, 2008

\begin{abstract}
We study in detail the optical forces generated by a plasmonic trap on a plasmonic nanoparticle. The permittivity of the trapped particle is tuned using a Drude model. The interplay between the plasmon resonances of the trap and of the particle can produce different regimes leading to attractive or repulsive forces. Hence a particle will be trapped or repulsed depending on its permittivity. Such a physical system should provide new functionalities for lab-on-the-chip applications. () 2008 Optical Society of America

OCIS codes: $240.6680,020.7010$.
\end{abstract}

Optical forces are known as momentum transfer between light and small objects. In the waist of a Gaussian beam, microsized objects can be trapped due to the transversal field gradients that create a stable trapping potential [1]. Ashkin and collaborators first pioneered this technique in the 1970 s by manipulating microsized particles in three dimensions [2,3]. Today, optical trapping and manipulation has become a widespread technique for chemical and biological analysis [4].

Over the past few years, there has been a growing interest in trapping metallic particles that support plasmon resonances. Indeed, the resonant excitation of the electrons at the plasmon resonance in such a particle creates a strong optical response. Chaumet et al. have studied the enhanced optical force between a metallic particle and a dielectric substrate under weak evanescent illumination [5]. Several authors have explained in terms of polarizability how metallic particles may be subjected to forces that attract them or push them away from the dielectric substrate [6,7]. The optical plasmonic forces in the presence of adjacent metallic particles have also been investigated by a variety of authors [8-11].

In addition to this work where plasmonic particles are trapped and manipulated, Quidant et al. [12], Volpe et al. [13], and Righini et al. [14] have proposed to use the field generated by a plasmonic structure to trap dielectric objects [12-14]. This elegant approach, which entirely relies on the strong field gradients generated by a plasmonic structure, provides a new way to integrate optical traps without the complex far-field optics used for optical tweezers. It has been recently used to trap biological entities [15], and to realize a $3 \mathrm{D}$ hybrid optical tweezer that combines a beam with a nanostructured substrate [16].

In this Letter, we study numerically the combination of the two preceding concepts and investigate the trapping of plasmonic nanoparticles using a plasmonic trap. Specifically, we explore effects related to tuning the plasmon resonance frequency of the trapped particle, with respect to the plasmon resonance frequency of the trap. Experimentally, continuous tuning of the plasmon resonance frequency of the trapped particle can be achieved using shell particles
[17-19]. Like this it is possible to cover the entire optical spectrum, from the visible to the infrared.

The system under study is shown in Fig. 1. It consists of a metallic patch, with a radius of $50 \mathrm{~nm}$ and a height of $40 \mathrm{~nm}$, lying upon a glass substrate (permittivity $\varepsilon=2.25$ ). We assume vacuum as cover material $(\varepsilon=1)$. A $p$-polarized plane wave illuminates the whole patch through the glass prism under total internal reflection (angle of incidence: $\theta=70^{\circ}$ ). The Green's tensor method is used to calculate the field distribution above the patch $[20,21]$. The optical force experienced by a metallic sphere located at a short distance above the patch is computed from this field distribution in the dipolar approximation [22].

To study the interaction between the patch and nanoparticles made with different plasmonic materials, we use Drude models to describe the permittivities of the patch and of the nanoparticles,

$$
\varepsilon(\omega)=1-\frac{\omega_{p}^{2}}{\omega^{2}+i \gamma \omega},
$$

where $\omega$ is the frequency, $\omega_{p}$ the plasma frequency, and $\gamma$ the damping factor [23]. In the following, we

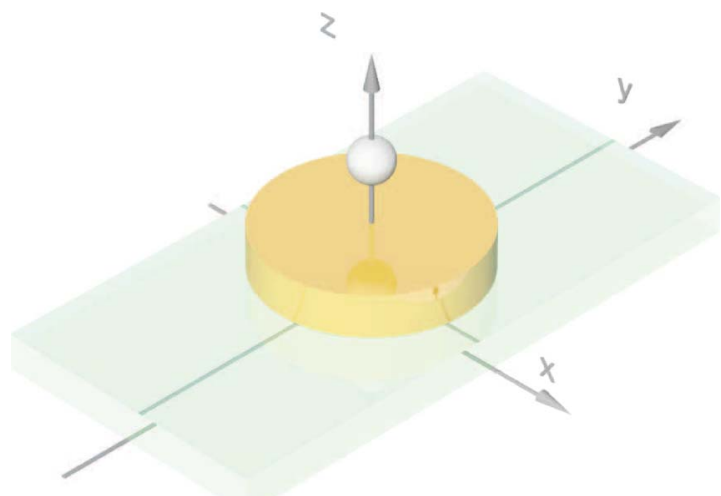

Fig. 1. (Color online) System under study: a cylindrical metallic patch is deposited on a glass substrate and illuminated under total internal reflection. The patch has a $50 \mathrm{~nm}$ radius and a $40 \mathrm{~nm}$ thickness; it is used to trap a metallic sphere with a $R=10 \mathrm{~nm}$ radius. Both metals are described with a Drude model using different plasma frequencies. 
keep the material of the patch constant and choose the following parameters to describe its metal: $\omega_{p}$ $=1 \mathrm{eV}$ and $\gamma=0.1 \mathrm{eV}$. The spectrum of the patch is shown in Fig. 2(a) as a continuous curve marked with dots. This figure indicates that for these parameters the plasmon resonance of the patch occurs at the frequency $\omega_{\text {patch }}=0.72 \mathrm{eV}$.

As a trapped particle, we use a small metallic sphere with a $R=10 \mathrm{~nm}$ radius and a permittivity given by Eq. (1). To investigate the interplay between the plasmon resonance of the trap and that of the trapped particle, we consider metallic spheres with a plasma frequency between $\omega_{p}=0.6$ and $1.8 \mathrm{eV}$; the absorption is kept constant: $\gamma=0.1 \mathrm{eV}$.

In the Rayleigh limit, the absorption cross-section $\sigma_{\text {abs }}$ of a small sphere with permittivity $\varepsilon(\omega)$ in an infinite homogeneous background $\varepsilon_{B}$ [23] is

$$
\sigma_{\text {abs }}(\omega)=4 k \pi R^{3} \Im\left\{\frac{\varepsilon(\omega)-\varepsilon_{B}}{\varepsilon(\omega)+2 \varepsilon_{B}}\right\},
$$

where $k$ is the wavenumber. The plasmon resonance condition for such a sphere occurs when the denominator in Eq. (2) vanishes, i.e., when $\omega_{\text {sphere }}=\omega_{p} / \sqrt{3}$. This is visible in Fig. 2(a), where the absorption cross section is shown for spheres described by a Drude model with $\omega_{p}=0.6 \ldots 1.8 \mathrm{eV}$. The corresponding plasmon resonance frequencies cover the range between $\omega_{\text {sphere }}=0.35$ and $1.04 \mathrm{eV}$, which includes the plasmon resonance frequency of the patch, around $\omega_{\text {patch }}$ $=0.72 \mathrm{eV}$.

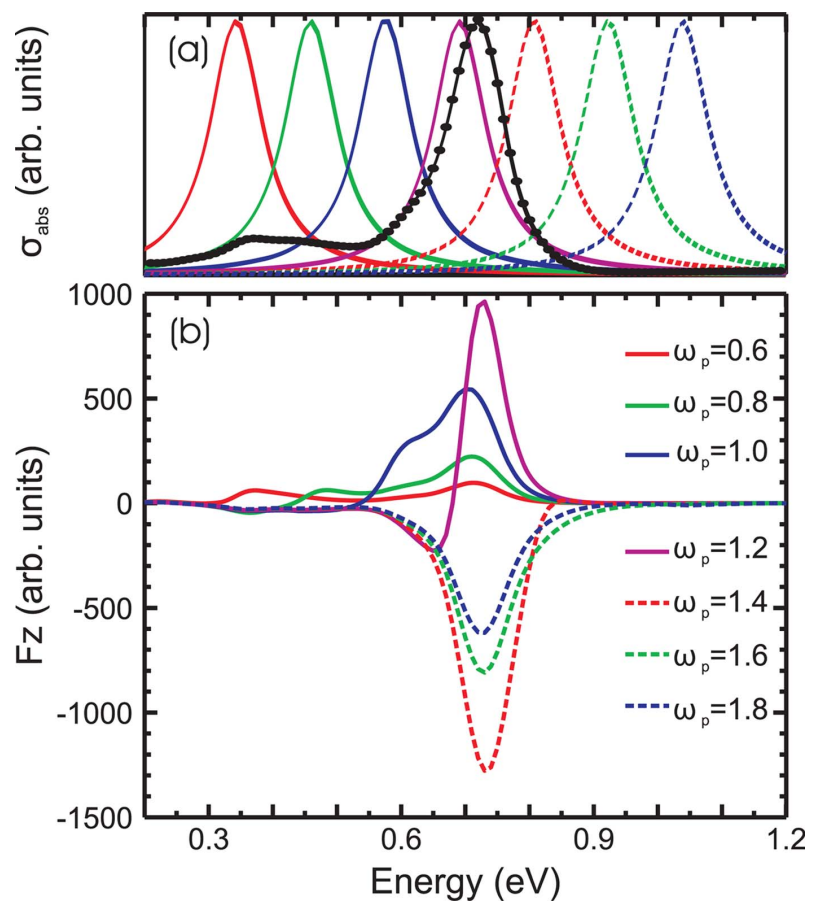

Fig. 2. (Color online) (a) Absorption cross section $\sigma_{\text {abs }}$ for $R=10 \mathrm{~nm}$ spheres described by a permittivity given by Eq. (1) with plasma frequencies between $\omega_{p}=0.6$ and $1.8 \mathrm{eV}$. The plasmon resonance of the trapping patch is shown as a solid curve with dots. (b) Spectra of the vertical force $F_{z}$ experienced by the different spheres.
The optical forces created by the patch on these nanoparticles are shown in Fig. 2(b). For clarity, we first concentrate on the vertical force, $F_{z}$ (Fig. 1), which attracts or repulses the particle toward the trap, depending whether it is negative or positive. All the forces reported here are calculated at a distance of $10 \mathrm{~nm}$ above the patch. We emphasize the behavior of the optical force and its change from attractive to repulsive, depending on the plasmonic conditions. The magnitude of the forces is of the order of piconewtons for standard illumination conditions; the interested reader is referred to [24] for a detailed discussion of the force magnitude and the competition with the van der Waals force.

Different regimes are observed in Fig. 2, depending on the relative positions of the resonance frequencies of the patch and the sphere. First, for $\omega_{\text {sphere }}<\omega_{\text {patch }}$, the force is repulsive. Two peaks are visible in the force spectrum: one corresponding to the plasmon resonance of the sphere and the other one to the plasmon resonance of the patch. The amplitude of this second peak increases as $\omega_{\text {sphere }}$ approaches $\omega_{\text {patch }}$. Second, for $\omega_{\text {sphere }}>\omega_{\text {patch }}$, only one peak is observed in the force spectrum, corresponding to the plasmon resonance of the patch. In this region, the force is always attractive and the nanoparticle moves toward the patch.

An interesting effect is observed in Fig. 2(b) for the sphere described by the Drude model with $\omega_{p}$ $=1.2 \mathrm{eV}$, plasmon resonance frequency of which is slightly smaller than that of the patch: depending on the illumination frequency, this particle will experience an attractive or a repulsive force. To examine when the force changes its sign from repulsive to attractive, we plot in Fig. 3 the vertical force $F_{z}$ as a function of the plasmon resonance frequency $\omega_{\text {sphere }}$ of the sphere, while keeping the illumination at $\omega$ $=0.72 \mathrm{eV}$. These calculations correspond to a collection of spheres with different Drude metals, described by different plasma frequencies. We clearly see that for $\omega_{\text {sphere }}<\omega_{\text {patch }}$, the amplitude of the re-

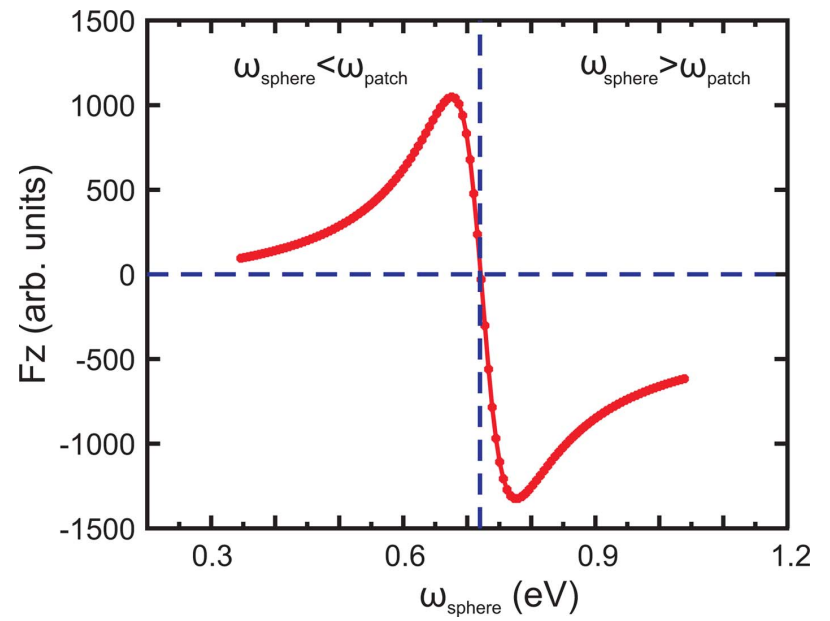

Fig. 3. (Color online) Vertical force $F_{z}$ experienced by a metallic sphere with $R=10 \mathrm{~nm}$ radius, as a function of its plasmon resonance frequency $\omega_{\text {sphere }}$ The vertical dashed line indicates the plasmon resonance frequency $\omega_{\text {patch }}$ of the trapping patch. 

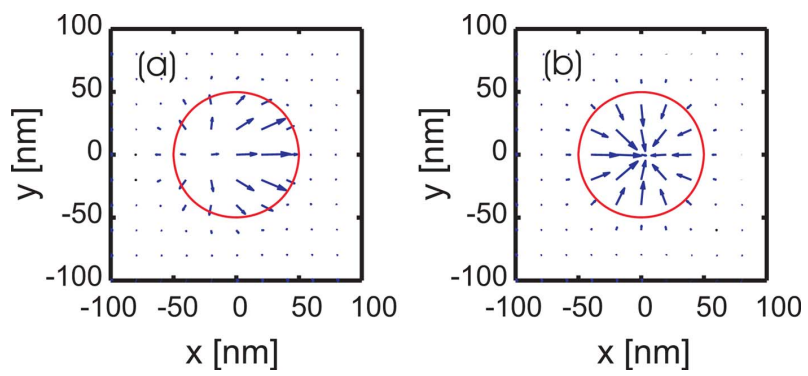

Fig. 4. (Color online) Vector plots of the horizontal force experienced by a metallic sphere located $10 \mathrm{~nm}$ above the patch. Two different Drude models are considered for the sphere: $\omega_{p}=$ (a) 1.2 and (b) $1.4 \mathrm{eV}$.

pulsive force $F_{z}$ increases with $\omega_{\text {sphere }}$ but suddenly decreases when it approaches $\omega_{\text {sphere }}$ (Fig. 3). For $\omega_{\text {sphere }}=\omega_{\text {patch }}$ the force vanishes: $F_{z}=0$. For $\omega_{\text {sphere }}$ $>\omega_{\text {patch }}$, the force $F_{z}$ becomes attractive with its magnitude increasing rapidly and then decreasing for larger values of $\omega_{\text {sphere }}$.

A similar behavior is observed for the horizontal optical force in the $x-y$ plane, as illustrated in Fig. 4. When the plasma frequency of the sphere $\left(\omega_{p}\right.$ $=1.2 \mathrm{eV}$ ) produces a plasmon frequency smaller than that of the trap, the particle is repulsed from the trap [Fig. 4(a)]. On the other hand, when the plasma frequency of the sphere $\left(\omega_{p}=1.4 \mathrm{eV}\right)$ creates a plasmon frequency larger than that of the trap, the particle is attracted toward the trap [Fig. 4(b)].

Since the strength and sign of the observed optical force depends on the relative spectral positions of the trap and of the trapped particle, this effect will change with the size of the trap, since the plasmon resonance depends on the trap geometry. This is illustrated in Fig. 5, where we now consider a larger patch with a radius of $R=100 \mathrm{~nm}$. The plasmon resonance of this patch is at $\omega_{\text {patch }}=0.79 \mathrm{eV}$. A similar be-

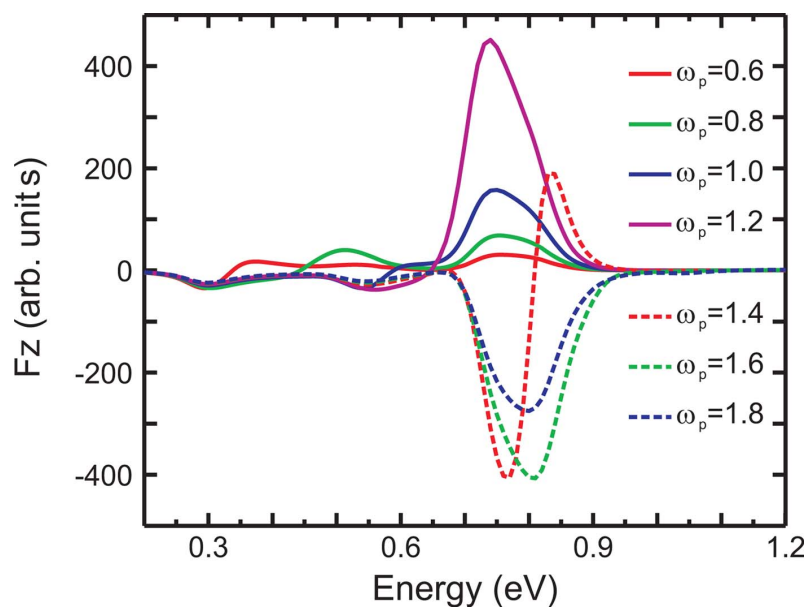

Fig. 5. (Color online) Force spectra experienced by different $R=10 \mathrm{~nm}$ radius metallic spheres with plasma frequencies between $\omega_{p}=0.6$ and $1.8 \mathrm{eV}$, for a larger patch with radius $100 \mathrm{~nm}$ and height $40 \mathrm{~nm}$. havior is observed in this case: the force is repulsive when $\omega_{\text {sphere }}<\omega_{\text {patch }}$ and attractive when $\omega_{\text {sphere }}$ $>\omega_{\text {patch }}$. However, this reversal occurs now at a larger frequency.

To summarize, we have discussed the trapping of a plasmonic particle in a plasmonic trap. The interplay between the plasmon resonances of the trap and of the particle can produce different regimes with attractive or repulsive forces. The integration of such systems should provide new functionalities for labon-the-chip applications.

Funding from the Swiss National Science Foundation (SNSF) (grant 200021-113735) is gratefully acknowledged.

\section{References}

1. D. G. Grier, Nature 424, 810 (2003).

2. A. Ashkin, Phys. Rev. Lett. 24, 156 (1970).

3. A. Ashkin, J. M. Dziedzic, J. E. Bjorkholm, and S. Chu, Opt. Lett. 11, 288 (1986).

4. M. P. MacDonald, G. C. Spalding, and K. Dholakia, Nature 426, 421 (2003).

5. P. C. Chaumet, A. Rahmani, and M. Nieto-Vesperinas, Appl. Opt. 45, 5185 (2006).

6. F. Claro and R. Rojas, Appl. Phys. Lett. 65, 2743 (1994).

7. J. R. Arias-Gonzalez and M. Nieto-Vesperinas, J. Opt. Soc. Am. A 20, 1201 (2003).

8. K. Halterman, J. M. Elson, and S. Singh, Phys. Rev. B 72, 075429 (2005).

9. B. Sepulveda, J. Alegret, and M. Käll, Opt. Express 15, 14914 (2007).

10. E. Lamothe, G. Lévêque, and O. J. F. Martin, Opt. Express 15, 9631 (2007).

11. A. S. Zelenina, R. Quidant, and M. Nieto-Vesperinas, Opt. Lett. 32, 1156 (2007).

12. R. Quidant, D. Petrov, and G. Badenes, Opt. Lett. 30, 1009 (2005).

13. G. Volpe, R. Quidant, G. Badenes, and D. Petrov, Phys. Rev. Lett. 96, 238101 (2006).

14. M. Righini, A. S. Zelenina, C. Girard, and R. Quidant, Nat. Phys. 3, 477 (2007).

15. X. Miao and L. Y. Lin, IEEE J. Sel. Top. Quantum Electron. 13, 1655 (2007).

16. A. N. Grigorenko, N. W. Roberts, M. R. Dickinson, and Y. Zhang, Nat. Photonics 2, 365 (2008).

17. S. J. Oldenburg, S. L. Westcott, R. D. Averitt, and N. J. Halas, J. Chem. Phys. 111, 4729 (1999).

18. J. B. Jackson and N. J. Halas, J. Phys. Chem. B 105, 2743 (2001).

19. H. Wang, D. W. Brandl, F. Le, P. Nordlander, and N. J. Halas, Nano Lett. 6, 827 (2006).

20. O. J. F. Martin and N. B. Piller, Phys. Rev. E 58, 3909 (1998).

21. M. Paulus and O. J. F. Martin, J. Opt. Soc. Am. A 18, 854 (2001).

22. P. C. Chaumet and M. Nieto-Vesperinas, Phys. Rev. B 61, $14119(2000)$.

23. C. F. Bohren and D. R. Huffman, Absorption and Scattering of Light by Small Particles (Wiley, 1983).

24. L. Novotny and C. Henkel, Opt. Lett. 33, 1029 (2008). 